\title{
Expression and clinical significance of cyclin kinase subunit 2 in colorectal cancer
}

\author{
MINHAO YU, MING ZHONG and ZHONGDONG QIAO \\ Department of Surgery, Renji Hospital, Shanghai Jiaotong University, Shanghai 200127, P.R. China
}

Received December 20, 2012; Accepted May 14, 2013

DOI: $10.3892 / 01.2013 .1456$

\begin{abstract}
The overexpression of cyclin kinase subunit 2 (CKS2) is tightly correlated with tumor aggressiveness and prognosis in various malignancies, including gastric, breast, liver and prostate cancer. However, whether CKS2 is upregulated in colorectal cancer (CRC) remains unknown. The aim of the present study was to analyze CKS2 expression levels in CRC, and to determine the clinical diagnostic and prognostic values of CKS2 overexpression in CRC patients. CKS2 expression was analyzed at the mRNA and protein levels by quantitative (q)PCR and western blot analysis. CKS2 expression was significantly upregulated in CRC compared with the adjacent non-cancer and normal colorectal tissues. The overexpression of CKS2 was correlated with poor differentiation and the pathological stage. In addition, CKS2 overexpression was correlated with aggressive tumor progression in $\mathrm{CRC}$, which indicated that CKS2 may serve as a good CRC biomarker.
\end{abstract}

\section{Introduction}

Colorectal cancer (CRC), one of the most common primary malignancies, demonstrates molecular heterogeneity during its development and progression (1). The prognosis of patients with CRC is commonly determined by traditional clinicopathological factors, including tumor grade and lymph node status $(2,3)$. Nevertheless, patients may have significantly different clinical outcomes despite the exhibition of similar clinicopathological features (4). Although serum carcinoembryonic antigen (CEA) has long been regarded as the most significant and common biomarker for CRC, there are limitations to the sole use of the CEA level for the early diagnosis and prognosis of CRC (5). Therefore, the identification of novel gene expression that is altered in CRC may aid the

Correspondence to: Professor Ming Zhong, Department of Surgery, Renji Hospital, Shanghai Jiaotong University, 1630 Dongfang Road, Shanghai 200127, P.R. China

E-mail: rjdrzhongming@163.com

Key words: cyclin kinase subunit 2, colorectal cancer biomarker understanding of the mechanisms of tumorigenesis, the development of diagnostic biomarkers, the prediction of the clinical prognosis and the design of targeted therapies.

The cyclin kinase subunit (CKS) proteins, which consist of CKS1 and CKS2 in vertebrates, are highly conserved molecules in eukaryotes. These proteins share $81 \%$ amino acid sequence homology (6). The overexpression of CKS1 has been demonstrated to be correlated with poor survival rates in patients with breast, colorectal, prostate and renal cancer (7-10). There is accumulating evidence that CKS2 expression, similar to that of CKS1, is upregulated in a variety of malignant tumors, including those of the prostate, bladder and liver (10-12). However, whether CKS2 is overexpressed in $\mathrm{CRC}$ remains unclear.

The present study aimed to show that CKS2 expression was significantly upregulated in $\mathrm{CRC}$, and that it was correlated with certain clinical features of CRC. The results suggested that the expression level of CKS2 may have a diagnostic and prognostic value for patients with CRC.

\section{Materials and methods}

Patients and specimens. As approved by the ethics committee of Renji Hospital (Shanghai Jiaotong University School of Medicine, Shanghai, China), colorectal cancer samples were obtained from 30 patients who underwent routine surgery for CRC at the Department of Surgery between 2010 and 2012. Patients were recruited immediately following surgery and samples of CRC, adjacent non-cancer and normal colorectal tissues were collected at that time. None of the patients had received any pre-operative treatment, including radiation or chemotherapy. Clinical data were recorded and the pathological classification was performed according to a staging system previously described (2). The tissues were immediately placed in TRIzol reagent for the extraction of RNA and protein. Written informed consent was obtained from all patients.

RNA extraction and quantitative (q)PCR analyses. Tissues were lysed and the total RNA was isolated using TRIzol reagent (Invitrogen Life Technologies, Carlsbad, CA, USA), according to the manufacturer's instructions. Following quantification of the RNA, a sample containing $2 \mu \mathrm{g}$ RNA was annealed to the oligo(dT) at $65^{\circ} \mathrm{C}$ for $5 \mathrm{~min}$ and cooled at $-4^{\circ} \mathrm{C}$ for $2 \mathrm{~min}$. A total volume of $20 \mathrm{ml}$ was used for the 
reverse transcription (RT) reaction; this contained RT-buffer, RNasin, reverse transcriptase, dNTPs and RNA-oligo(dT) mixtures. The PCR reaction was conducted at $42^{\circ} \mathrm{C}$ for $60 \mathrm{~min}$, and the following primers were used: CKS2 forward, 5'-GCTCTTCGCGCTCTCGTTTCATTT-3' and reverse, 5'-ACTCTGTTGGACACCAAGTCTCCT-3'. The PCR reactions were terminated subsequent to 35 cycles. For the PCR quantitation, the SYBR, primers and cDNA were mixed, and the reaction was performed for 40 cycles using the MJ Research PTC-100 Thermal Cycler system (Bio-Rad, Hercules, CA, USA). The data were normalized with the glyceraldehyde3-phosphate dehydrogenase (GAPDH) housekeeping gene. All primers were custom-synthesized by Sangon Biotech (Shanghai) Co., Ltd. (Shanghai, China).

Western blot analysis. The total protein was extracted from $\sim 0.5 \mathrm{~g}$ frozen tissue using radioimmunoprecipitation assay (RIPA) buffer (Beyotime, Shanghai, China). Aliquots containing $30 \mathrm{mg}$ protein were subjected to sodium dodecyl sulfate-polyacrylamide gel electrophoresis and electroblotted onto a polyvinylidene difluoride membrane (Amersham Biosciences AB, Uppsala, Sweden) for western blot analyses for $2 \mathrm{~h}$. Following incubation with 5\% skimmed milk for $2 \mathrm{~h}$, the membranes were incubated with the primary antibody [anti-CKS2, dilution of 1:3,000 in Tris-buffered saline and $0.1 \%$ Tween 20 (TBST)] for $1 \mathrm{~h}$ at room temperature. Each membrane was then washed three times with TBST for $10 \mathrm{~min}$ followed by incubation with the secondary antibody goat antimouse IgG-HRP (Santa Cruz Biotechnology Inc., Santa Cruz, CA, USA) (1:10,000-30,000 dilution) for $1 \mathrm{~h}$. Following three 10-min washes with TBST, the specifically bound antibodies were detected with the Enhanced Chemiluminescence (ECL) kit (MultiScience Biotech Co., Shanghai, China), according to the manufacturer's instructions. The intensity of the bands was quantified usign the Tanon GIS system (Tanon, Shanghai, China) and the data were normalized to the GAPDH loading controls.

Statistical analysis. All data were processed with SPSS 13.0 software (SPSS, Inc., Chicago, IL, USA). The Kruskal-Wallis non-parametric test was used to analyze the correlations between CKS2 mRNA expression and various clinicopathological features. $\mathrm{P}<0.05$ was considered to indicate a statistically significant difference, and was calculated by the two-tailed test.

\section{Results}

Correlations between CSK2 expression and clinicopathological features. The clinical findings are summarized in Table I. A total of 14 males (46.7\%) and 16 females (53.3\%), with ages ranging between 27 and 81 years (median, 62 years; mean, 58.7 years) were recruited into the study. Thirteen patients presented with rectal cancer and 17 with colon cancer. The post-operative pathological classifications were performed according to the NCCN Guidelines Version 2.2012, and included 15 patients $(50.0 \%)$ each in stages I and II.

Expression of CKS2 is elevated at the mRNA and protein levels in $C R C$. To determine whether CKS2 was overexpressed in CRC, the mRNA and protein levels of CKS2 were measured
Table I. Correlations between CKS2 expression and clinicopathological features in CRC.

\begin{tabular}{lcc}
\hline Characteristics & No. of patients & $\begin{array}{c}\text { CKS2 protein } \\
\text { expression }\end{array}$ \\
\hline Age (years) & & \\
$<50$ & 22 & $0.152 \pm 0.013$ \\
$\geq 50$ & & $0.201 \pm 0.024$ \\
Gender & 16 & \\
Female & 14 & $0.178 \pm 0.019$ \\
Male & & $0.192 \pm 0.032$ \\
Tumor diameter $(\mathrm{cm})$ & 12 & $0.138 \pm 0.026$ \\
$<4$ & 18 & $0.214 \pm 0.010^{\mathrm{a}}$ \\
$\geq 4$ & & \\
Differentiation & 4 & $0.165 \pm 0.021$ \\
Well & 19 & $0.169 \pm 0.019$ \\
Moderate & 7 & $0.237 \pm 0.027^{\mathrm{a}}$ \\
Poor & & \\
Location & 13 & $0.182 \pm 0.016$ \\
Rectum & 17 & $0.202 \pm 0.014$ \\
Colon & & $0.116 \pm 0.051$ \\
pTNM stage & 15 & \\
II & & \\
III & & \\
\hline
\end{tabular}

${ }^{\mathrm{a}} \mathrm{P}<0.05$. CKS2 protein expression is presented as the mean \pm standard deviation. CKS2, cyclin kinase subunit 2; CRC, colorectal cancer; pTNM, pathological tumor node metastasis.

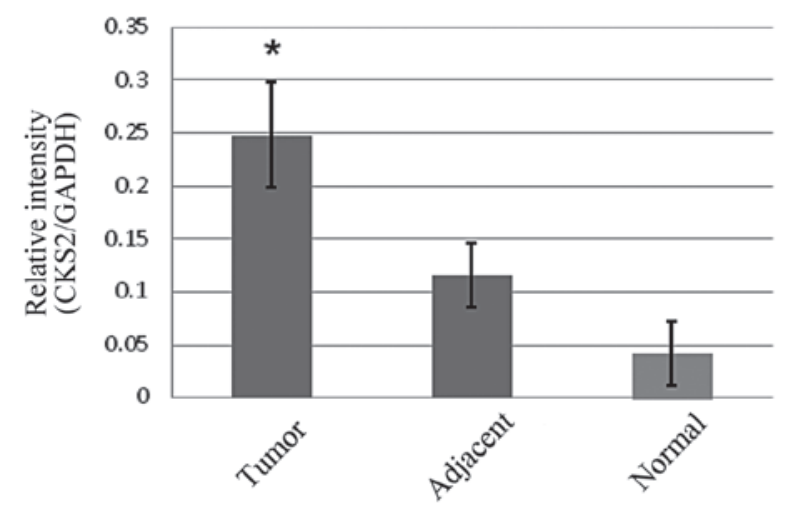

Figure 1. Overexpression of cyclin kinase subunit 2 (CKS2) at the mRNA level in different types of tissue. Total RNA samples were extracted from colorectal cancer (CRC), adjacent non-cancer and normal colorectal tissues. Expression of CKS2 was assessed by quantitative (q)PCR. Data were normalized to glyceraldehyde-3-phosphate dehydrogenase (GAPDH). ${ }^{*} \mathrm{P}<0.05$ vs. control.

in the tumor and adjacent non-tumor tissues, as well as in the normal colorectal tissue. qPCR analyses revealed that the mRNA levels of CKS2 were significantly increased in the $\mathrm{CRC}$ tissue compared with the adjacent non-tumor and normal 


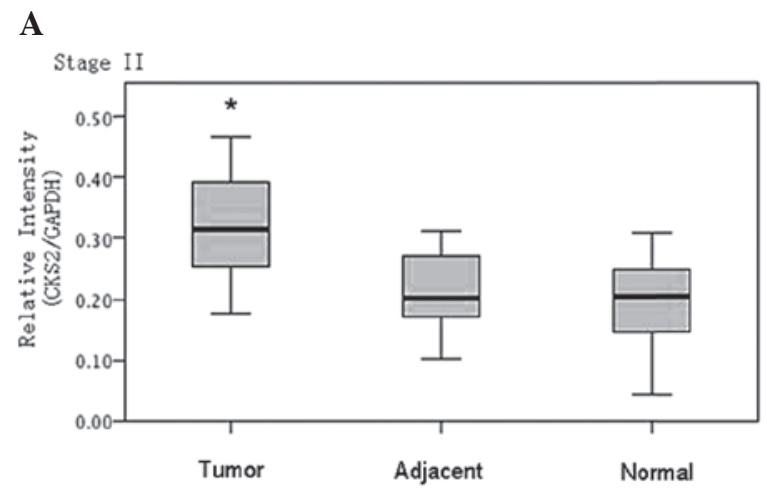

B

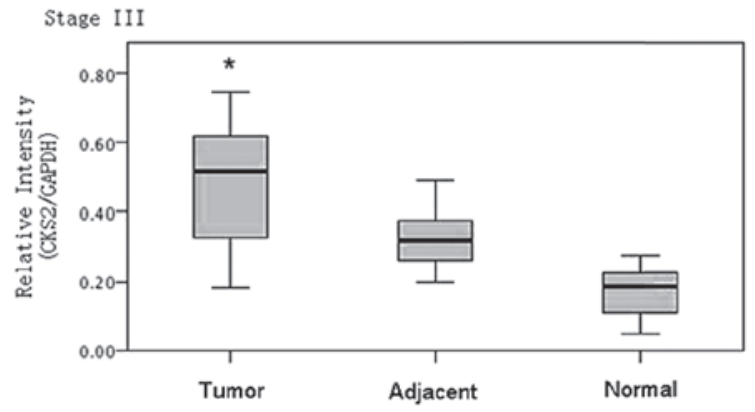

C

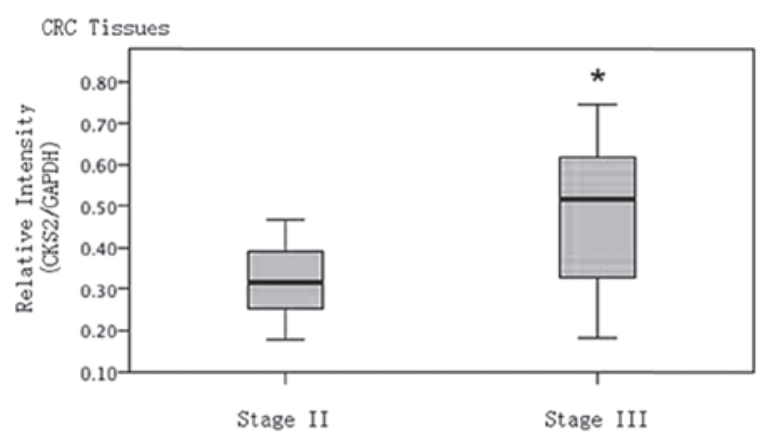

D

CKS2 expression level

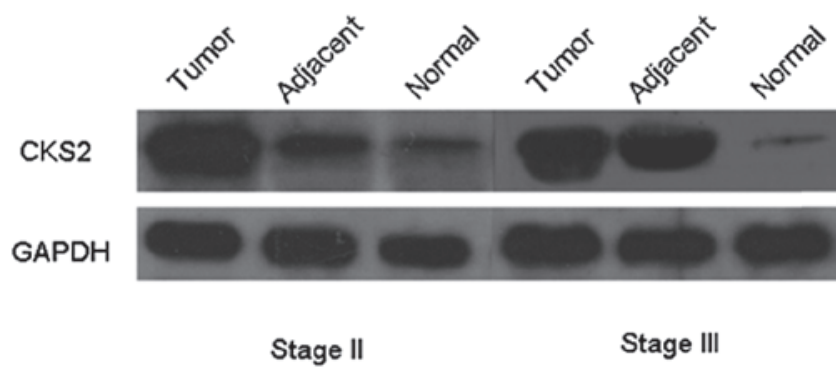

Figure 2. Western blot analyses were used to determine cyclin kinase subunit 2 (CKS2) protein expression levels in tumor tissues from stage II and III colorectal cancer patients and adjacent non-cancer and normal colorectal tissues. (A) In stage II CRC, CKS2 protein levels were significantly increased compared with the normal and adjacent tissues. ${ }^{~} \mathrm{P}<0.05$ vs. control. (B) In stage III CRC, CKS2 protein levels were significantly increased in the tumor and adjacent tissues compared with the normal tissue. ${ }^{\text {" }} \mathrm{P}<0.05$ vs. control. (C) Tumor tissues from stage III CRC patients demonstrated significantly higher CKS2 protein levels than those from stage II patients. ${ }^{*} \mathrm{P}<0.05$ vs. control. (D) The proteins extracted from the tissues were subjected to western blot analyses as indicated. Glyceraldehyde-3-phosphate dehydrogenase (GAPDH) was used as the loading control. colorectal tissues (Fig. 1). Western blot analyses demonstrated that the expression of the CKS2 protein was also upregulated in the CRC tissue samples, as shown in Fig. 2.

Overexpression of CKS2 is correlated with the aggressive behavior of $C R C$. To further examine the clinicopathological relevance of CKS2 overexpression in CRC, CKS2 expression was analyzed in correlation with pathological features of tumors. The results revealed that the overexpression of CKS2 at the protein level was significantly correlated with tumor size, differentiation and pathological tumor node metastasis (pTNM) stage (Table I). No significant correlation was detected between CKS2 overexpression and other clinicopathological features, such as patient age and gender or tumor location.

\section{Discussion}

The CKS proteins, including CKS1 and CKS2, are essential components of cyclin/cyclin-dependent kinase (CDK) complexes that are involved in the regulation of cell cycle progression. The CKS proteins exhibit $81 \%$ amino acid sequence identity (13). The dysregulation of the CKS proteins and other cell cycle-related regulators, including the cyclins and CDKs, has been demonstrated to be associated with several types of tumors $(14,15)$.

The present study identified that CKS2 was overexpressed at the mRNA and protein levels in CRC tissues in comparison with the adjacent non-cancer and normal colon tissues. However, the opposite was observed in certain samples. This may occur in clinical practice due to the lack of a clear definition of the adjacent non-tumor tissue. Furthermore, these data clearly demonstrated that the overexpression of CKS2 was significantly correlated with tumor differentiation and lymph node metastasis, which may have contributed to the development of CRC. However, as the complete course of the CRC patients was not available, a Kaplan-Meier survival analysis could not be conducted.

Although the level of CKS2 expression was significantly higher in the tumor tissue than in the adjacent non-cancer and normal colorectal tissues, there were certain discrepancies in the correlation between CKS overexpression at the mRNA and protein levels and lymph node metastasis. These discrepancies may be attributed to the relatively small sample size in the present study. Different translation efficiencies or stabilities of the protein in the tumor tissues may also have caused the discrepancies in the results. In addition, genetic and epigenetic factors, including DNA methylation, genetic mutation and abnormal post-transcriptional regulation, may have contributed to the variation in the results (16). Further studies are required to clarify this issue.

In conclusion, to the best of our knowledge, this is the first study to demonstrate that CKS2 is overexpressed in CRC. The results suggested that the aberrant expression of CKS2 may contribute to the development and progression of CRC, and that CKS2 expression patterns may be of diagnostic and prognostic value for CRC patients.

\section{References}

1. Hanahan D and Weinberg RA: The hallmarks of cancer. Cell 100: $57-70,2000$. 
2. Edge SB: AJCC Staging Manual. 7th edition. Springer, New York, NY, 2010.

3. Le Voyer TE, Sigurdson ER, Hanlon AL, et al: Colon cancer survival is associated with increasing number of lymph nodes analyzed: a secondary survery of intergroup trial INT-0089. J Clin Oncol 21: 2912-2919, 2003.

4. Siena S, Sartore-Bianchi A, Di Nicolantonio F, et al: Biomarkers predicting clinical outcome of epidermal growth factor receptortargeted therapy in metastatic colorectal cancer. J Natl Cancer Inst 101: 1308-1324, 2009.

5. Chu DZ, Erickson CA, Russell MP, et al: Prognostic significance of carcinoembryonic antigen in colorectal carcinoma. Serum levels before and after resection and before recurrence. Arch Surg 126: 314-316, 1991.

6. Williams RT, Wu L, Carbonaro-Hall DA, et al: Identification of a novel cyclin-like protein in human tumor cells. J Biol Chem 268 8871-8880, 1993

7. Slotky M, Shapira M, Ben-Izhak O, et al: The expression of the ubiquitin ligase subunit Cks1 in human breast cancer. Breast Cancer Res 7: R737-R744, 2005.

8. Shapira M, Ben-Izhak O, Linn S, et al: The prognostic impact of the ubiquitin ligase subunits Skp2 and Cks1 in colorectal carcinoma. Cancer 103: 1336-1346, 2005.

9. Lan Y, Zhang Y, Wang J, et al: Aberrant expression of Cks1 and $\mathrm{Cks} 2$ contributes to prostate tumorigenesis by promoting proliferation and inhibiting programmed cell death. Int J Cancer 123: 543-551, 2008.
10. Liu Z, Fu Q, Lv J, Wang F and Ding K: Prognostic implication of p27Kip1, Skp2 and Cks1 expression in renal cell carcinoma: a tissue microarray study. J Exp Clin Cancer Res 27: 51, 2008.

11. Kawakami K, Enokida H, Tachiwada T, et al: Identification of differentially expressed genes in human bladder cancer through genome-wide gene expression profiling. Oncol Rep 16: 521-531, 2006.

12. Kang MA, Kim JT, Kim JH, et al: Upregulation of the cycline kinase subunit CKS2 increases cell proliferation rate in gastric cancer. J Cancer Res Clin Oncol 135: 761-769, 2009.

13. Shen DY, Fang ZX, You P, et al: Clinical significance and expression of cyclin kinase subunits 1 and 2 in hepatocellular carcinoma. Liver Int 30: 119-125, 2009.

14. Alhasan SA, Ensley JF, Sarkar FH, et al: Genistein induced molecular changes in a squamous cell carcinoma of the head and neck cell line. Int J Oncol 16: 333-338, 2000.

15. Hansel DE, Dhara S, Huang RC, et al: CDC2/CDK1 expression in esophageal adenocarcinoma and precursor lesions serves as a diagnostic and cancer progression marker and potential novel drug target. Am J Surg Pathol 29: 390-399, 2005.

16. Martinsson-Ahlzén HS, Liberal V, Grünenfelder B, et al: Cyclin-dependent kinase-associated proteins Cks1 and Cks2 are essential during early embryogenesis and for cell cycle progression in somatic cells. Mol Cell Biol 28: 5698-5709, 2008. 